\title{
Home Parenteral Nutrition: How Well Are Scottish Patients Monitored?
}

\author{
N. S. Hallum ${ }^{1}$, R. F. McKee ${ }^{1}$ and J. P. Baxter ${ }^{2}$ \\ ${ }^{1}$ Scottish Home Parenteral Nutrition Managed Clinical Network, Glasgow Royal Infirmary, Glasgow UK and ${ }^{2}$ Ninewells \\ Hospital, Dundee UK
}

The provision of Home Parenteral Nutrition (HPN) allows patients with intestinal failure to leave hospital and gain independence. The successful management of these patients necessitates regular multiprofessional review and monitoring in clinics or on an inpatient basis during subsequent admissions. The Scottish HPN Managed Clinical Network (MCN) was set up to ensure both equity of access and high standards of care for all its patients. Now in its seventh year, audit is undertaken to establish the adequacy of monitoring of its patients. All enrolled centres collect an agreed dataset on an ongoing basis using a standard purpose-built database, which is combined yearly for review. The current audit assesses frequency and content of review in 2006. Standards set out by the HPN MCN advise that all patients are reviewed by a multidisciplinary nutrition team on a three- to four-monthly basis. Review should ideally comprise weight, anthropometry, blood tests and vitamin/micronutrient measurements. A figure of $100 \mathrm{~d}$ was used as the frequency with which review should be undertaken. Data is available for fifty-three patients in 141 clinic appointments in seven centres.

\begin{tabular}{|c|c|c|c|c|c|}
\hline \multicolumn{6}{|c|}{ Frequency of patient review } \\
\hline \multicolumn{3}{|c|}{ Always reviewed within $100 \mathrm{~d}$} & Sometimes & \multicolumn{2}{|c|}{ Never reviewed within $100 \mathrm{~d}$} \\
\hline 18 & & 18 & & 17 & \\
\hline \multicolumn{6}{|c|}{ HPN reviews in 2006} \\
\hline \multicolumn{3}{|l|}{ Review type } & $<100 \mathrm{~d}$ since last review & \multicolumn{2}{|c|}{$>100$ days since last review } \\
\hline \multicolumn{2}{|c|}{ Outpatient (OPD) } & \multirow{2}{*}{\multicolumn{2}{|c|}{$\begin{array}{l}85 \\
94\end{array}$}} & \multicolumn{2}{|l|}{56} \\
\hline \multirow{2}{*}{\multicolumn{6}{|c|}{$\begin{array}{l}\text { OPD }+ \text { admission } \\
\text { Adequacy of HPN reviews }\end{array}$}} \\
\hline & & & & & \\
\hline Component & Weight & Anthropometry & Routine blood tests & Micronutrients & Haematinics \\
\hline Yes & 121 & 34 & 131 & 88 & 70 \\
\hline No & 20 & 107 & 10 & 53 & 71 \\
\hline
\end{tabular}

The Scottish HPN MCN patients are not being reviewed as often as is recommended by the network protocols, with only eighteen out of fifty-three patients meeting the criteria on every visit throughout 2006. However patients reviewed less frequently did not appear to have more admissions and complications.

During patient reviews, weight measurement and routine blood tests are successfully taken but anthropometry and micronutrient levels are less well recorded. Full blood count, urea and electrolytes and liver function tests were sent at $89 \%$ of reviews. Other tests were less often requested: $\mathrm{Mg} \mathrm{65 \%}$; C reactive protein 58\%; glucose $46 \%$ of visits. Micronutrients and haematinics were often omitted. Bone biochemistry is not well documented (Ca $72 \%$; vitamin D $28 \%$ ).

There may be some informal review taking place which is not being recorded. Nevertheless there is clear room for improvement in our HPN review. Anthropometry could be improved by better organisation and staff training. Many factors impinge on our ability to achieve complete blood results including organisational difficulties with requests, a central trace element laboratory and poor venous access.

The frequency and content of review were set by the MCN on the basis of expert opinion rather than any higher level of evidence. Although it is clear that we should improve some aspects of our review, consideration should be given to studies which examine the outcome in HPN patients reviewed less frequently. 